PROCEEDINGS OF THE

AMERICAN MATHEMATICAL SOCIETY

Volume 128, Number 10, Pages 3081-3089

S 0002-9939(00)05356-9

Article electronically published on March 2, 2000

\title{
STABLE MAPS OF POLISH SPACES
}

\author{
A. V. OSTROVSKY
}

(Communicated by Alan Dow)

\begin{abstract}
We define the notions of stable and transquotient maps and study the relation between these classes of maps. The class of stable maps contains all closed and open maps and their compositions. The transquotient maps preserve the property of being a Polish space, and every stable map between separable metric spaces is transquotient.

In particular, a composition of closed and open maps (the intermediary spaces may not be metric) preserves the property of being a Polish space. This generalizes the results of Sierpiński and Vainstein for open and closed maps.
\end{abstract}

In [5] the author introduced a concept of system $\left(\eta_{y}\right)_{y \in Y}$ associated to a map $f$ : $X \rightarrow Y$ with the help of which we give here new classes of stable and transquotient $\operatorname{maps} 1$

All the maps in this paper are continuous and onto. All the spaces are regular.

Let $f: X \rightarrow Y$ be a map from a separable, complete metrizable (= Polish) space $X$ onto a metric space $Y$. Then $Y$ is also Polish in the following cases:

a) if $f$ is an open or a closed map [7], [9];

b) if $f$ is a compact-covering map or an $s$-covering man [2], [3], [5];

c) if $f$ is a composition of open and perfect maps [3].

First, we will show that the class of stable maps contains the above-mentioned classes. Second, we obtain from Theorem 0 all the above-mentioned statements a) - c) and some new results. In particular, a composition of closed and open maps preserves the property of being a Polish space.

Notice, that in all the cases a)-c) there is a closed subset $X_{0} \subset X$ such that the restriction $f \mid X_{0}$ is a perfect map onto $Y$ (see [2], [3], [5]).

Theorem 0. Let $f: X \rightarrow Y$ be a stable map from a Polish space $X$ onto a metrizable space $Y$. Then:

(1) There is a countable set $Y_{\sigma} \subset Y$ and a Polish space $X_{0} \subset X$ such that the restriction $f \mid X_{0}$ is a perfect map onto $Y \backslash Y_{\sigma}$.

(2) $Y$ is a Polish space.

Received by the editors October 27, 1997 and, in revised form, November 5, 1998.

1991 Mathematics Subject Classification. Primary 54C10, 54D18.

Key words and phrases. Polish space, stable map, transquotient map, point-harmonious map.

${ }^{1}$ The system, which was studied in [5, Lemmas 1-3], was triquotient.

${ }^{2} \mathrm{~A}$ map $f: X \rightarrow Y$ is compact-covering (resp. $s$-covering or countable-compact-covering) if every compact (resp. countable compact) $K \subset Y$ is the image of some compact $B \subset X$.

(C)2000 American Mathematical Society 


\section{Stable AND TRANSQUOtient MAPS}

Our approach will be to establish a characterization of stable, transquotient and harmonious maps in terms of transmittable systems and to give a relation between these classes of maps. We start first with some general notation:

A system associated to a map $f: X \rightarrow Y$ is a family $\left(\eta_{y}\right)_{y \in Y}$ where each $\eta_{y}$ is a family of open subsets of $X$ satisfying the condition $U \cap f^{-1}(y) \neq \varnothing$ for all $U \in \eta_{y}$. The system is said to be transmittable if the following condition holds:

(a) for every $U \in \eta_{y}$ there is a neighborhood $O(y)$ of $y$ such that $U \in \eta_{y^{\prime}}$ for every $y^{\prime} \in O(y)$.

It is clear that if $U \in \eta_{y}$, then $y \in \operatorname{Int} f(U)$. A map may have many transmittable systems and a system is called trivial if $\eta_{y}=\{X\}$ for every $y \in Y$.

Definition 1. The map $f: X \rightarrow Y$ is stable if it admits a transmittable system satisfying the following condition:

(b) If $U \in \eta_{y}$ and $V$ is open in $X$ such that $V \supset U \cap f^{-1}(y)$, then $V \in \eta_{y}$.

Definition 2. A map $f: X \rightarrow Y$ is transquotient if it admits the transmittable system satisfying the following condition:

(c) If $U \in \eta_{y}$ and $\gamma=\left\{U_{\alpha}\right\}_{\alpha \in A}$ is a family of open subsets of $X$ such that $\bigcup_{\alpha \in A} U_{\alpha} \supset f^{-1}(y) \cap U$, then there is a finite number $U_{\alpha_{1}}, \ldots, U_{\alpha_{n}} \in \gamma$ and an open $O(y)$ such that $\bigcup_{i=1}^{n} U_{\alpha_{i}} \in \eta_{y^{\prime}}$ for every $y^{\prime} \in O(y) \backslash\{y\}$.

Remark 0. Let $\eta_{y}$ be a transquotient family for $f$. Define a new family for $f$ :

$\tilde{\eta}_{y}=\left\{V: V\right.$ is open in $X$ and $\exists U \in \eta_{y}$ such that $\left.V \supset U \cap f^{-1}(y)\right\}$.

In particular $X \in \tilde{\eta}_{y}$. Notice that the transmittable property holds for $\tilde{\eta}_{y}$ since for $V \in \tilde{\eta}_{y}$ we can consider $\gamma=\{V\}$ in (c) and then, obviously, $V \in \tilde{\eta}_{y^{\prime}}$ for every $y^{\prime} \in O(y)$. Since every cover of $f^{-1}(y) \cap V$ is the cover of $f^{-1}(y) \cap U$, we have by (c) that $\tilde{\eta}_{y}$ is a transquotient family for $f$.

If $V$ is open in $X$ and there is $U \in \tilde{\eta}_{y}$ such that $V \supset f^{-1}(y) \cap U$ (in particular, if $\left.V \supset f^{-1}(y)\right)$, then, obviously, $V$ is also an element of $\tilde{\eta}_{y}$. Hence, a transquotient map $f$ is stable.

It should be noted that the following lemma uses an idea of A.H. Stone's proof [8, Lemma 1].

Lemma 1. Let $f: X \rightarrow Y$ be a map. If $Y$ is first-countable and if every fiber $f^{-1}(y)$ is hereditarily Lindelöf, then the following conditions are equivalent:

(i) $f$ is stable;

(ii) $f$ is transquotient.

Proof. By Remark 0 (ii) $\Rightarrow$ (i). We will show (i) $\Rightarrow$ (ii). Suppose the family $\eta_{y}$ satisfies Definition 1 and does not satisfy Definition 2. This means that (c) fails for some $U \in \eta_{y}$ and family $\gamma=\left\{U_{i}\right\}_{i \in \omega}$. It is obvious that there is an open decreasing base $\left\{O_{n}(y)\right\}_{n \in \omega}$ at $y$ such that for every $\gamma_{n}=\bigcup_{i=0}^{n} U_{i}$ there is a $y_{n}^{\prime} \in$ $O_{n}(y) \backslash O_{n+1}(y)$, for which $\gamma_{n} \notin \eta_{y_{n}^{\prime}}$ and, hence, $M_{n}=\left(U \cap f^{-1}\left(y_{n}^{\prime}\right)\right) \backslash \gamma_{n} \neq \varnothing$ (we assume that $O_{0}(y)$ is a neighbourhood of $y$ such that $U \in \eta_{y^{\prime}}$ for each $\left.y^{\prime} \in O_{0}(y)\right)$.

Let $M=\bigcup\left\{M_{n}: n \in \omega\right\}$. Now let us show that $f^{-1}(y) \cap U \cap \operatorname{cl}_{X} M \neq \varnothing$. Suppose the contrary, then $U^{\prime}=U \backslash \operatorname{cl}_{X} M \supset f^{-1}(y) \cap U$ and $U^{\prime} \in \eta_{y}$. By the transmittable property, for some $n$ we have $U^{\prime} \in \eta_{y_{n}^{\prime}}$. By our construction, $\gamma_{n}$ $\supset \gamma_{n} \cap U \cap f^{-1}\left(y_{n}^{\prime}\right)=U^{\prime} \cap f^{-1}\left(y_{n}^{\prime}\right)$ and, hence, $\gamma_{n} \in \eta_{y_{n}^{\prime}}$. That is impossible. 
Choose $x \in f^{-1}(y) \cap U \cap \mathrm{cl}_{X} M$; then $x$ belongs to some $\gamma_{n}$. It is clear that $x \in$ $T=f^{-1}(y) \cap U \cap \operatorname{cl}_{X}\left(\bigcup\left\{M_{k}: k>n\right\}\right)$. Taking into consideration that $M_{k} \subset U \backslash \gamma_{n}$ for $k>n$, and hence $T \cap \gamma_{n}=\varnothing$, we get a contradiction with $x \in \gamma_{n}$.

Let us consider some examples of stable and transquotient maps.

Proposition A. Open maps and closed maps $f: X \rightarrow Y$ are stable.

For an open map: $\eta_{y}=\{U: U$ is open and $y \in f(U)\}$.

For a closed map: $\eta_{y}=\left\{U \subset X: U\right.$ is open in $X$, and $\left.U \supset f^{-1}(y)\right\}$. It is a well known fact that for every $y \in Y$ and every open $U \supset f^{-1}(y)$ there is an open $O(y)$ such that $f^{-1}(O(y)) \subset U$.

Every open map with $\eta_{y}=\{U: U$ is open and $y \in f(U)\}$ is transquotient but the space $Y=R \bmod Z$, obtained by identifying the integers $Z$ in the real line $R$ to one point $y$, gives a closed and non-transquotient map $f: R \rightarrow Y$. Indeed, let $\gamma_{1}$ be a cover of $f^{-1}(y)$ by open intervals of length $<1 / 2$. Then for every finite subcover $\gamma_{2}$ of $\gamma_{1}$ and every open $O(y)$ there is $y^{\prime} \in O(y) \backslash\{y\}$ such that $\bigcup \gamma_{2} \cap f^{-1}\left(y^{\prime}\right)=\varnothing$.

Proposition B. Suppose $f: X \rightarrow Y$ and $\varphi: Y \rightarrow Z$ are stable maps. Then the composition $g=\varphi \circ f: X \rightarrow Z$ is also a stable map.

Let $\eta_{y}(f)$ and $\eta_{z}(\varphi)$ be stable families for $f$ and $\varphi$, respectively. Let us define $\eta_{z}(g)=\left\{U: \exists T \in \eta_{z}(\varphi) \forall y \in T \cap \varphi^{-1}(z)\left(U \in \eta_{y}(f)\right)\right\}$ and let us show that $g$ is a stable map. It is clear that $X \in \eta_{z}(g)$ for every $z \in Z$.

(a) Let us assume $U \in \eta_{z}(g)$ and $y \in T \cap \varphi^{-1}(z)$. By (a) for $f$ there is an open $O(y) \subset T$ such that $U \in \eta_{y^{\prime}}(f)$ for each $y^{\prime} \in O(y)$. Let $T^{\prime}=\bigcup\{O(y): y \in$ $\left.T \cap \varphi^{-1}(z)\right\}$. Obviously, $T^{\prime} \supset T \cap \varphi^{-1}(z)$. Hence, $T^{\prime} \in \eta_{z}(\varphi)$.

We choose (according to (a) for $\varphi$ ) an open $O(z)$ such that $T^{\prime} \in \eta_{z^{\prime}}(\varphi)$ for every $z^{\prime} \in O(z)$, and then we have $U \in \eta_{z^{\prime}}(g)$.

(b) Suppose $V \supset U \cap g^{-1}(z)$ for an open $V$, where $U \in \eta_{z}(g)$. Then $V \in \eta_{y}(f)$ for every $y \in T \cap \varphi^{-1}(z)$ and, hence, $V \in \eta_{z}(g)$.

\section{Proof of Theorem 0}

A map $f: X \rightarrow Y$ from a metric space $X$ is uniformly sequentially complete if each $f^{-1}(S)$ is complete with the given metric on $X$ for every convergent, including its limit, sequence $S=\{y\} \cup \bigcup\left\{y_{i}: y_{i} \rightarrow y\right\}$ in $Y$.

Proposition C. If $f: X \rightarrow Y$ is transquotient with appropriate families $\eta_{y}, Y_{0} \subseteq$ $Y, Z=\bigcup_{\alpha \in A} U_{\alpha}$ and $U_{\alpha} \in \eta_{y}$ for each $y \in f\left(U_{\alpha}\right) \backslash Y_{0}$, then $f \mid\left(Z \backslash f^{-1}\left(Y_{0}\right)\right)$ : $Z \backslash f^{-1}\left(Y_{0}\right) \rightarrow f(Z) \backslash Y_{0}$ is transquotient.

The new transquotient families are defined as $\tilde{\eta}_{y}=\left\{U \backslash f^{-1}\left(Y_{0}\right) \subseteq Z \backslash f^{-1}\left(Y_{0}\right)\right.$ : $\left.U \in \eta_{y}\right\}$.

Lemma 2. Let $f: X \rightarrow Y$ be a transquotient, uniformly sequentially complete map between separable metric spaces $X, Y$. Then there exists a $G_{\delta}$-set $X^{\prime} \subset X$ and $a$ countable set $Y_{\sigma} \subset Y$ such that the restriction $f \mid X^{\prime}$ is a perfect map onto $Y \backslash Y_{\sigma}$.

Proof. There is a perfect extension $f^{*}: X^{*} \rightarrow Y$ of $f$ and $Z \subset X^{*}$ such that $Z \supset X$, every $f^{-1}(S)$ is closed in $Z$ for every convergent, including its limit sequence $S \subset Y$ and $Z=\bigcap_{i \in \omega} O_{i}$, where $O_{i}$ are open in $X^{*}$.

Indeed, suppose $B_{Y} \supset Y$ and $B_{\tilde{X}}$ are compact spaces containing $Y$ and the completion $\tilde{X}$ of $X$, respectively. Then $\tilde{X}$ is a $G_{\delta}$-set in $B_{\tilde{X}}$ and the first coordinate 
projection $\pi$ on $B_{Y} \times B_{\tilde{X}}$ is a perfect map. Let $X^{*}=\pi^{-1}(Y), Z=Y \times \tilde{X}$ and $f^{*}=\pi \mid X^{*}$. Then $f^{*}: X^{*} \rightarrow Y$ is also perfect. Finally, we can identify the graph $G \subset X^{*}$ of the map $f$ with $X$ and the projection of $G$ with $f$; then $f^{*}$ is the extension of $f$.

Notice that $f^{*-1}(S)$ is always compact for a convergent sequence $S$ including its limit because $f^{*-1}(S)=S \times B_{\tilde{X}}$ and this is a product of two compact sets.

Note also that by the construction of the transmittable system constructed in Remark $0\left(\tilde{\eta}_{y}\right)$, we can assume that there exists a transmittable system $\eta_{y}$ that makes $f$ both transquotient and stable.

Let us choose for each $y \in Y$ some $U_{0}^{y} \in \eta_{y}, U_{0}^{y} \subset X$ and for each point $x \in U_{0}^{y} \cap f^{-1}(y)$ a neighborhood $U(x) \subset U_{0}^{y}$ such that $c l_{X^{*}} U(x) \subset O_{0}$. According to Definition 2 there are finitely many $U\left(x_{i}\right), i=1,2, \ldots, n$, and a neighborhood $O(y)$ such that $U_{1}^{y}=\bigcup_{i=1}^{n} U\left(x_{i}\right) \in \eta_{\xi}$ for every $\xi \in O(y) \backslash\{y\}$.

Hence, $\delta_{1}=\{O(y): y \in Y\}$ is an open cover of $Y$ and for every $y \in Y$ we have $f\left(U_{1}^{y}\right) \supset O(y)$.

Since every separable metric space is a Lindelöf space and every Lindelöf space is paracompact, we can consider the countable, locally finite open refinement $\gamma_{1}=$ $\left\{U_{k}\right\}_{k \in \omega}$ of $\delta_{1}$. We can choose for every $U_{k} \in \gamma_{1}$ some $y_{k}$ such that $O\left(y_{k}\right) \supset U_{k}$.

Denote $\delta_{1}\left(\gamma_{1}\right)=\left\{O\left(y_{k}\right): k \in \omega\right\}$ and $Y_{0}=\left\{y_{k}: k \in \omega\right\}$.

Choose for every $U_{k} \in \gamma_{1}$ the corresponding elements $O\left(y_{k}\right) \in \delta_{1}\left(\gamma_{1}\right), U_{1}^{y_{k}} \in \eta_{y_{k}}$ and denote $W_{1, k}=U_{1}^{y_{k}} \cap f^{*-1}\left(U_{k}\right) \subset X$. By our construction

(*) $W_{1, k} \in \eta_{y}$ for every point $y \in U_{k} \backslash Y_{0}$.

In fact, for every $y^{\prime} \in O\left(y_{k}\right) \backslash\left\{y_{k}\right\}$ (see the definition $\tilde{\eta}_{y}$ ) we obtain $(*$ ). Let us denote

$$
X_{0}=\left(\bigcup_{k \in \omega} W_{1, k}\right) \backslash f^{-1}\left(Y_{0}\right), \quad f_{0}=f \mid X_{0}: X_{0} \rightarrow Y \backslash Y_{0} .
$$

According to $(*)$ and Proposition $\mathrm{C} f_{0}$ is transquotient. Let us denote

$$
X_{0}^{*}=\left(c l_{X^{*}} \bigcup_{k \in \omega} W_{1, k}\right) \backslash f^{*-1}\left(Y_{0}\right), \quad f_{0}^{*}=f^{*} \mid X_{0}^{*}: X_{0}^{*} \rightarrow Y \backslash Y_{0} .
$$

It is clear that $f_{0}^{*}$ is a perfect map. Since $\left\{W_{1, k}\right\}_{k \in \omega}$ is a locally finite family, we have: $X_{0}^{*}=\left(\bigcup_{k \in \omega} c l_{X^{*}} W_{1, k}\right) \backslash f^{*-1}\left(Y_{0}\right)$ and, hence, $X_{0} \subset X_{0}^{*} \subset O_{0}$.

We may consider $O_{1}, X_{0}, X_{0}^{*}, f_{0}, f_{0}^{*}$ instead of $O_{0}, X, X^{*}, f, f^{*}$ and repeat the construction. In this way we obtain a countable subspace $Y_{1} \subset\left(Y \backslash Y_{0}\right)$ and:

a perfect map $f_{1}^{*}: X_{1}^{*} \rightarrow Y \backslash\left(Y_{0} \cup Y_{1}\right)$, where $X_{1}^{*} \subset O_{1}$ and $X_{1} \subset X_{1}^{*} \subset X_{0}^{*}$;

a transquotient map $f \mid X_{1}$ onto $Y \backslash\left(Y_{0} \cup Y_{1}\right)$, where $X_{1} \subset X_{0}$.

Thus, we have the subspaces $O_{i} \supset X_{i}^{*} \supset X_{i+1}^{*}, i \in \omega$, where $X_{i}^{*} \supset X_{i}$ is closed in $X^{*} \backslash \bigcup_{k=0}^{i} f^{*-1}\left(Y_{k}\right)$ and every $f_{i}^{*}: X_{i}^{*} \rightarrow Y \backslash \bigcup_{k=0}^{i} Y_{k}$ is perfect. Denote $Y_{\sigma}=\bigcup_{i \in \omega} Y_{i}$. Evidently, $Y_{\sigma}$ is countable. It is obvious that $F=\bigcap_{i \in \omega} X_{i}^{*} \subset Z$ is closed in $X^{*} \backslash f^{*-1}\left(Y_{\sigma}\right)$ and, hence, $f^{*} \mid F$ is a perfect map onto $Y \backslash Y_{\sigma}$. Denote $X^{\prime}=F \cap X$. We shall prove that $f^{\prime}=f \mid X^{\prime}$ is a perfect map onto $Y^{\prime}=Y \backslash Y_{\sigma}$.

By inspection of the construction of the spaces $X_{n}^{*}$ one can see that they are of the form $X_{n}^{*}=T_{n} \backslash f^{*-1}\left(\bigcup_{i=0}^{n} Y_{n}\right)$, where the sets $T_{n}$ are closed in $X^{*}, T_{0} \supseteq T_{1} \supseteq \ldots$ and $T_{n} \subseteq O_{n}$. Let $T=\bigcap_{n} T_{n}$; then $T \subset Z$, because $T_{n} \subset O_{n}$. Let $S$ be a sequence contained in $Y^{\prime}$ and assume that $S$ is convergent and contains its own limit. $f^{-1}(S)$ is closed in $Z$. Hence $f^{-1}(S) \cap T$ is closed in $T$, because $T \subset Z$. Hence $f^{-1}(S)=$ $f^{-1}(S) \cap F=f^{-1}(S) \cap T$ is closed in $f^{*-1}(S) \cap T$. But the set $f^{*-1}(S)$ is compact. Thus $f^{\prime-1}(S)$ is compact. 
To prove that $f^{\prime}\left(X^{\prime}\right)=Y \backslash Y_{\sigma}$, note first that for every $y \in Y \backslash Y_{\sigma}$ the set $L_{n}=$ $c l_{X^{*}} f^{-1}(y) \cap X_{n}^{*}$ is compact $(n \in \omega)$. By our construction $L_{n} \supset L_{n+1}$ and $L_{n} \neq \varnothing$ since $X_{n}^{*} \supset X_{n}$ and $f^{-1}(y) \cap X_{n} \neq \varnothing$. Hence, there is $x \in \bigcap_{n} L_{n}=c l_{X^{*}} f^{-1}(y) \cap F$. Since $f^{-1}(y)$ is closed in $Z$ and $F \subset Z$, we have $x \in f^{-1}(y) \cap F=f^{\prime-1}(y)$.

To verify that $f^{\prime}(A)$ is closed in $Y^{\prime}$ for every closed $A \subset X$, note that if $y_{i}=$ $f^{\prime}\left(x_{i}\right), x_{i} \in A$, and $y_{i} \rightarrow y$, then, as $C=f^{\prime-1}\left(\left\{y_{i}: i \in \omega\right\} \cup\{y\}\right)$ is compact, there is a limit point $x$ for the sequence $\left\{x_{i}: i \in \omega\right\}$ of the points of $C$. But then $x \in A$ because $x_{i}$ are in $A$ and $A$ is closed. Obviously, $f^{\prime}(x)=y \in f^{\prime}(A)$.

Since $f \mid X^{\prime}$ is a perfect map onto $Y \backslash Y_{\sigma}, X^{\prime}$ is a closed subset of $X \backslash f^{-1}\left(Y_{\sigma}\right)$, hence $X^{\prime}$ is a $G_{\delta}$-set in $X$.

Let $X$ be a topological space. The strong Choquet game $C$ is played as follows. Players $\alpha, \beta$ take turns, with $\beta$ playing first, choosing nonempty open subsets of $X$. Player $\beta$ also plays a point in his open set at each move and $\alpha$ must play an open set containing this point. Each open set played by any player is contained in the opponent's previous move

$\begin{array}{lllll}\beta & U_{0}, x_{0} & U_{1}, x_{1} & \cdots & \\ \alpha & & V_{0} & & U_{1}\end{array}$

where $x_{i} \in U_{i}, x_{i} \in V_{i}$ and $U_{0} \supseteq V_{0} \supseteq U_{1} \supseteq V_{1} \supseteq \ldots$. We say that $\alpha$ wins a run of this game $U_{0}, V_{0}, V_{1}, \ldots$ if $\bigcap \begin{aligned} & n \\ & n\end{aligned}\left(=\bigcap U_{n}\right) \neq \varnothing$. One can require without losing generality that at every step the first player plays a different point. We say that $X$ is a strong Choquet space if $\alpha$ has a winning strategy in the strong Choquet game $C$ for $X[1$.

Lemma 3. Let $f: X \rightarrow Y$ be a transquotient map of a strong Choquet space $X$ onto $Y$. Then $Y$ is also a strong Choquet space.

Proof. Denote by $\Sigma$ a winning strategy for $\alpha$ in $C$. Denote by $C^{\prime}$ the strong Choquet game on $Y$ and by $\alpha^{\prime}, \beta^{\prime}$ its players. We will describe a winning strategy for $\alpha^{\prime}$ in $C^{\prime}$.

Step 0. Let $\beta^{\prime}$ start with $\left(U_{0}^{\prime}, y_{0}\right)$. For every $x_{0} \in f^{-1}\left(y_{0}\right)$ take an open $U_{0} \subset$ $f^{-1}\left(U_{0}^{\prime}\right), x_{0} \in U_{0}$. Let $\beta$ play $\left(U_{0}, x_{0}\right)$, then $\alpha$ answers, using $\Sigma$, by $f_{0}\left(\left(x_{0}, U_{0}\right)\right)=$ $V_{0} \subseteq U_{0}, x_{0} \in V_{0}$.

By Remark 0 to Definition 2 we may suppose $X \in \eta_{y_{0}}$. Since the elements $V_{0}$ cover $X \cap f^{-1}\left(y_{0}\right)$, there is a finite family $\gamma_{0}$ of elements $V_{0}$ and an open $V_{0}^{\prime} \subseteq$ $U_{0}^{\prime}, y_{0} \in V_{0}^{\prime}$ such that $\bigcup \gamma_{0} \in \eta_{y^{\prime}}$ for every $y^{\prime} \in V_{0}^{\prime} \backslash\left\{y_{0}\right\}$. Then $\alpha^{\prime}$ answers by $f_{0}^{\prime}\left(\left(y_{0}, U_{0}^{\prime}\right)\right)=V_{0}^{\prime} \subset U_{0}^{\prime}, y_{0} \in V_{0}^{\prime}$.

Step 1. Let $\beta^{\prime}$ play $\left(U_{1}^{\prime}, y_{1}\right)$, where $y_{1} \in U_{1}^{\prime} \subseteq V_{0}^{\prime}$.

Then $\beta$ plays $\left(U_{1}, x_{1}\right)$, where $U_{1} \subset V_{0} \cap f^{-1}\left(U_{1}^{\prime}\right)$ for every $V_{0} \in \gamma_{0}$ and every $x_{1} \in V_{0} \cap f^{-1}\left(y_{1}\right)$. Then $\alpha$, using $\Sigma$, answers by $f_{1}\left(\left(x_{0}, U_{0}\right),\left(x_{1}, U_{1}\right)\right)=V_{1} \subset U_{1}$, $x_{1} \in V_{1}$.

By the definition of transquotient maps, there is a finite family $\gamma_{1}$ of elements $V_{1}$ and an open $V_{1}^{\prime} \subseteq U_{1}^{\prime}, y_{1} \in V_{1}^{\prime}$ such that $\bigcup \gamma_{1} \in \eta_{y^{\prime}}$ for every $y^{\prime} \in V_{1}^{\prime} \backslash\left\{y_{1}\right\}$. Then $\alpha^{\prime}$ answers by $f_{1}^{\prime}\left(\left(y_{0}, U_{0}^{\prime}\right),\left(y_{1}, U_{1}^{\prime}\right)\right)=V_{1}^{\prime} \subset U_{1}^{\prime}, y_{1} \in V_{1}^{\prime}$. By construction $U_{1}^{\prime} \supset f\left(\bigcup \gamma_{1}\right) \supset V_{1}^{\prime}$.

Step $n+1$. Suppose we defined a map $f_{n}^{\prime}\left(\left(y_{0}, U_{0}^{\prime}\right), \ldots,\left(y_{n}, U_{n}^{\prime}\right)\right)=V_{n}^{\prime} \subset U_{n}^{\prime}$, $y_{n} \in V_{n}^{\prime}$ for $\left(y_{0}, U_{0}^{\prime}\right), \ldots,\left(y_{n}, U_{n}^{\prime}\right)$ and finite families $\gamma_{n}$ of open sets $V_{n}$ such that $\bigcup \gamma_{n} \in \eta_{y^{\prime}}$ for every $y^{\prime} \in V_{n}^{\prime}$ and $U_{n}^{\prime} \supset f\left(\bigcup \gamma_{n}\right) \supset V_{n}^{\prime}$. Let $\beta^{\prime}$ play $\left(U_{n+1}^{\prime}, y_{n+1}\right)$. 
Then $\beta$ plays $\left(U_{n+1}, x_{n+1}\right)$ for every $V_{n} \in \gamma_{n}$ and every $x_{n+1} \in V_{n} \cap f^{-1}\left(y_{n+1}\right)$ with $U_{n+1} \subset V_{n} \cap f^{-1}\left(U_{n+1}^{\prime}\right)$ and $\alpha$ answers, using $\Sigma, f_{n+1}\left(\left(x_{0}, U_{0}\right), \ldots,\left(x_{n+1}, U_{n+1}\right)\right)$ $=V_{n+1} \subset U_{n+1}, x_{n+1} \in V_{n+1}$.

By the definition of transquotient maps there is a finite family $\gamma_{n+1}$ of elements $V_{n+1}$ and an open $V_{n+1}^{\prime} \subset U_{n+1}^{\prime}, y_{n+1} \in V_{n+1}^{\prime}$ such that $\bigcup \gamma_{n+1} \in \eta_{y^{\prime}}$ for every $y^{\prime} \in V_{n+1}^{\prime} \backslash\left\{y_{n+1}\right\}$. Then $\alpha^{\prime}$ answers $f_{n+1}^{\prime}\left(\left(y_{0}, U_{0}^{\prime}\right), \ldots,\left(y_{n+1}, U_{n+1}^{\prime}\right)\right)=V_{n+1}^{\prime} \subset$ $U_{n+1}^{\prime}, y_{n+1} \in V_{n+1}^{\prime}$.

By our construction

(*) $U_{n+1}^{\prime} \supset f\left(\bigcup \gamma_{n+1}\right) \supset V_{n+1}^{\prime}$.

By König's theorem an infinite, finitely branching tree must have an infinite branch and we obtain in this way a sequence $V_{0}^{*} \supset V_{1}^{*} \supset \ldots \supset V_{i}^{*} \supset \ldots$, obtained according to a winning strategy for $\alpha$, where $V_{i}^{*} \in \gamma_{i}$. Since $\alpha$ wins, there is $x \in$ $\bigcap_{n} V_{n}^{*}$ and, hence, $x \in \bigcup \gamma_{n}$ for every $n \in \omega$. Since by definition of strong Choquet game $V_{n}^{\prime} \supset U_{n+1}^{\prime}$, we have according to $(*): f(x) \in \bigcap_{n} V_{n}^{\prime}$, and $\alpha^{\prime}$ wins.

Proof of Theorem 0. Lemma 2 implies part (1) of Theorem 0 . Notice that $X$ is completely metrizable if and only if $X$ is a metrizable strong Choquet space [1] and we obtain from Lemmas 1 and 3 part (2) of Theorem 0 (see also [4, Corollary $7.3])$.

\section{REMARKS}

We begin by recalling the following definition [6]:

Definition 3. A map $f: X \rightarrow Y$ is harmonious if one can assign to every compact $K \subset Y$ a family $\eta_{K}$ of open subsets of $X$ satisfying the following conditions:

$\left(a^{\prime}\right)$ If $U \in \eta_{K}$, then there exists a compact $B \subset U$ such that $f(B)=K$ and for every open $V \supset B$ we have $V \in \eta_{K}$.

$\left(b^{\prime}\right)$ If $U \in \eta_{K}$, then there exists an open $O(K)$ such that $U \in \eta_{K^{\prime}}$ for every compact $K^{\prime} \subset O(K)$.

We obtain the definition of a point-harmonious map by replacing $K$ and $K^{\prime}$ in the above definition by points $y$ and $y^{\prime}$.

By Theorems 1 and 2 from [6] in case of separable metrizable spaces $X, Y$ the class of harmonious (resp. point-harmonious) maps coincides with the class of compact-covering (resp. $s$-covering) maps.

Recall that triquotient maps may be defined as transquotient maps satisfying condition (c) from Definition 2 for every $y^{\prime} \in O(y)$.

Proposition D. Transquotient maps, triquotient maps, point-harmonious maps and harmonious maps are stable.

Let $f: X \rightarrow Y$ be a map. Then 1) $\Rightarrow 2) \Rightarrow 3) \Rightarrow 4) \Rightarrow 5$ ).

1) $f$ is harmonious.

2) $f$ is point-harmonious.

3) $f$ is triquotient.

4) $f$ is transquotent.

5) $f$ is stable.

1) $\Rightarrow 2) \Rightarrow 3$ ) follow from the definition of harmonious and point-harmonious maps.

$3) \Rightarrow 4) \Rightarrow 5$ ) follow from the definition of transquotient maps and Remark 0 . 
Proposition E. Compact-covering maps and s-covering maps onto a metrizable space are stable.

The corresponding stable family $\eta_{y}$ is constructed in Example 1.

Proposition F. If $f: X \rightarrow Y$ is a stable map with compact fibres, then $f$ is pointharmonious.

Let $\eta_{y}$ be a stable family for $f$ and let $\varepsilon_{y}=\left\{B=c l_{X} U \cap f^{-1}(y): U \in \eta_{y}\right\}$. Define a transmittable family for $f$ as follows:

$\eta_{y}^{\prime}=\left\{U^{\prime}: U^{\prime}\right.$ is open in $X$ and there is $B \in \varepsilon_{y}$ such that $\left.U^{\prime} \supset B\right\}$.

It is clear that the condition $\left(a^{\prime}\right)$ is satisfied. Let us consider $U^{\prime} \in \eta_{y}^{\prime}$ and some compact $B \in \varepsilon_{y}, B \subset U^{\prime}$. Since $X$ is regular, there is an open set $V \supset B$ such that $c l_{X} V \subset U^{\prime}$. Since $B \supset U \cap f^{-1}(y)$ where $U \in \eta_{y}$, there is an open $O(y)$ such that $V \in \eta_{y^{\prime}}$ for every $y^{\prime} \in O(y)$. Since $U^{\prime} \supset B^{\prime}=c l_{X} V \cap f^{-1}\left(y^{\prime}\right)$ and $B^{\prime} \in \varepsilon_{y^{\prime}}$, we have $U^{\prime} \in \eta_{y^{\prime}}^{\prime}$ for every $y^{\prime} \in O(y)$ and $\left(b^{\prime}\right)$ is also satisfied.

A map $f: X \rightarrow Y$ is feebly open if for every (non-empty) open $V \subset X$ we have $\operatorname{Int} f(V) \neq \varnothing$.

Recall that a space $Y$ is an $F_{\mathrm{II}}$-space if there is no closed subset of $Y$ which is of the first category in itself. The following theorem shows that part (2) of Theorem 0 can be extended onto a larger class of $F_{\mathrm{II}^{-}}$-spaces.

Theorem 1. Let $f: X \rightarrow Y$ be a transquotient map. If $X$ is an $F_{\mathrm{II}}-$ space, then $Y$ is also an $F_{\mathrm{II}}$-space.

We need the following lemma.

Lemma 4. Let $f: X \rightarrow Y$ be a transquotient map. Then there is a closed $Z \subset X$ such that the restriction $f \mid Z$ is a feebly open, transquotient map onto $Y$.

Proof . Suppose $f$ is not feebly open; then there is a non-empty open $V \subset X$ such that $\operatorname{Int} f(V)=\varnothing$. Denote $X_{1}=X \backslash V, f_{1}=f \mid X_{1}, \eta_{y}^{1}=\left\{U^{1}=U \cap X_{1}: U \in \eta_{y}\right\}$. First, $U^{1} \cap f_{1}^{-1}(y) \neq \varnothing$. Suppose not; then for some $U \in \eta_{y}, V \supset U \cap f^{-1}(y)$. According to Remark 0, we may suppose that $f$ is not only transquotient but also stable with respect to $\eta_{y}$, hence there is an open $O(y)$ such that $f(V) \supset O(y)$, which is impossible.

It is clear that the transmittable property (a) for $f_{1}$ is satisfied.

Let $U^{1} \in \eta_{y}^{1}$ and $\gamma^{1}=\left\{U_{\alpha}^{1}\right\}$ be an open cover of $f_{1}^{-1}(y) \cap U^{1}$. Let us take an open $U \in \eta_{y}$ such that $U \cap X_{1}=U^{1}$. Obviously, $\gamma=\left\{T_{\alpha}^{1}=U_{\alpha}^{1} \cup\left(X \backslash X_{1}\right)\right\}$ is an open cover of $U \cap f^{-1}(y)$. Hence, there exist $T_{\alpha_{1}}^{1}, \ldots, T_{\alpha_{n}}^{1}$ and $O(y) \subset Y$ such that $T^{1}=\bigcup_{i=1}^{n} T_{\alpha_{i}}^{1} \in \eta_{y^{\prime}}$ for every $y^{\prime} \in O(y) \backslash\{y\}$. It is clear that $T^{1} \cap X_{1}=\bigcup_{i=1}^{n} U_{\alpha_{i}}^{1} \in$ $\eta_{y^{\prime}}^{1}$ for every $y^{\prime} \in O(y) \backslash\{y\}$ and, hence, (c) is also satisfied and $f_{1}$ is transquotient.

Suppose we obtained for some $\alpha$ a strictly decreasing sequence of closed subsets $X_{\beta} \subset X(\beta<\alpha)$ and transquotient maps $f_{\beta}=f \mid X_{\beta}$ onto $Y$ with hereditary transquotient family $\eta_{y}^{\beta}=\left\{U^{\beta}=U \cap X_{\beta}: U \in \eta_{y}\right\}$.

If $\alpha$ is not a limit ordinal, that is, if $\alpha=\beta+1$, and $f \mid X_{\beta}$ is not feebly open, we may consider $X_{\alpha}, X_{\beta}$ instead of $X_{1}, X$ and repeat the construction above.

For the limit ordinal $\alpha$ define: $X_{\alpha}=\bigcap_{\beta<\alpha} X_{\beta}, f_{\alpha}=f \mid X_{\alpha}, \eta_{y}^{\alpha}=\left\{U^{\alpha}=\right.$ $\left.U \cap X_{\alpha}: U \in \eta_{y}\right\}$ and prove that for every $y \in Y$ and every $U^{\alpha} \in \eta_{y}$ we have $U^{\alpha} \cap f_{\alpha}^{-1}(y) \neq \varnothing$.

Suppose the contrary. Then for some $U \in \eta_{y}$ and $U^{\alpha}=U \cap X_{\alpha}$, we have $U \cap X_{\alpha} \cap f^{-1}(y)=\varnothing$. It is clear that $\left\{O_{\beta}=X \backslash X_{\beta}\right\}_{\beta<\alpha}$ is an open cover of 
$f^{-1}(y) \cap U$. Since $X_{\beta} \supset X_{\beta+1}$ and $f$ is transquotient, there is $\beta<\alpha$ and $y^{\prime} \in O(y)$ such that $O_{\beta} \in \eta_{y^{\prime}}$ and, hence, $O_{\beta} \cap X_{\beta} \in \eta_{y^{\prime}}^{\beta}$. This contradicts the condition $\varnothing \notin \eta_{y^{\prime}}^{\beta}$ because $O_{\beta} \cap X_{\beta}=\varnothing$.

Just like for $X_{1}$ we can prove that $f \mid X_{\alpha}$ is a transquotient map with hereditary transquotient family. We may repeat the above construction if $f \mid X_{\alpha}$ is not feebly open, etc. Since $\left\{X_{\alpha}\right\}$ is a strictly decreasing sequence of closed sets, there is $\alpha_{0}$ such that $Z=X_{\alpha_{0}}=X_{\alpha_{0}+1}=\ldots$. Let us denote $f_{\alpha_{0}}=f \mid Z$. Then $f_{\alpha_{0}}$ is a feebly open, transquotient map onto $Y$.

To prove Theorem 1 suppose the contrary and let $Y_{0} \subset Y$ be a closed subspace of the first category and, hence, $Y_{0}$ can be represented as a countable union of nowhere dense and closed in $Y$ subsets $F_{i}$. It is not hard to prove (see Proposition C) that $f_{0}=f \mid X_{0}$, where $X_{0}=f^{-1}\left(Y_{0}\right)$, is a transquotient map. By Lemma 4 we can suppose that $f_{0}$ is feebly open and, hence, every $f^{-1}\left(F_{i}\right)$ is a closed nowhere dense subset. Then $X_{0}$ is a subspace of the first category in itself, which is impossible by definition of $F_{\mathrm{II}}$-space.

Let $X$ be a (non-separable) complete metric space, and let $Y$ be a metric space. I don't know whether the map $f$ in the following cases 1 and 2 must be transquotient (or $Y$ completely metrizable):

1. $f: X \rightarrow Y$ is a countable-inductively perfect map (i.e., for every countable closed $Y_{0} \subset Y$ there is $X_{0} \subset X$ such that $f \mid X_{0}$ is a perfect map onto $Y_{0}$ ).

2. $f: X \rightarrow Y$ is a composition of closed and open maps (the domain and the range of all maps are regular).

\section{EXAMPLES}

The assumption that $X$ is separable cannot be omitted in Theorem 0:

Example 1. A stable, non-transquotient map $f: X \rightarrow Y$ from a completely metrizable space $X$ onto metric space $Y$ which does not satisfy conditions (1) and (2) of Theorem 0 .

In fact, let $Y$ not be a union of a countable set and an absolutely $G_{\delta}$-set. For example, $Y=\mathbb{Q}^{\omega}$ (where $\mathbb{Q}$ is the space of rational numbers) and let $X$ be a topological sum of all compact subsets of $Y$ with the obvious map $f: X \rightarrow Y$. It is clear that $X$ is completely metrizable and, by Lemma $3, f$ is not transquotient. Since a perfect image of a completely metrizable space is completely metrizable, $f$ does not satisfy the conditions of Theorem 0 .

We'll prove that $f$ is stable. In fact, let us define:

$\eta_{y}=\{U: U$ is open in $X$ and for every compact $K \subset Y$ containing $y$, there is a compact $B \subset X$ such that $f^{-1}(y) \cap B \subset U$ and $\left.f(B)=K\right\}$.

Obviously, the condition (b) of Definition 1 is fulfilled. We will verify the transmittable property (a). Let us assume the contrary: for some $U \in \eta_{y}$ there is a base $\left\{O_{i}\right\}$ at the point $y$, points $y_{i} \in O_{i}$ and compacts $K_{i} \subset O_{i}$, which contain $y_{i}$ such that there is no compact subset $B_{i}$ of $X$ satisfying the conditions: $f^{-1}\left(y_{i}\right) \cap B_{i} \subset U$ and $f\left(B_{i}\right)=K_{i}$. It is clear that $S=\{y\} \cup \bigcup\left\{K_{i}: i \in \omega\right\}$ is a compact set and $y \in S$. By definition of $\eta_{y}$ there is a compact $B \subset X$ for which $f(B)=S$ and $f^{-1}(y) \cap B \subset U$. Then compact $B \backslash U$ contains a closed countable discrete family of non-empty sets $\left(f^{-1}\left(y_{i}\right) \cap B\right) \backslash U$. This is impossible. 
Example 2. A transquotient (and stable) map $f: X \rightarrow Y$ from a countable, Polish space $X$ onto a compact, metric space $Y$ and the following conditions are satisfied:

1) $f: X \rightarrow Y$ is not $s$-covering (= not point-harmonious) map.

2) $f=f_{2} \circ f_{1}$, where $f_{1}$ is a closed map and $f_{2}$ is an open map 3

Let $p_{0}<p_{1}<\ldots<p_{n}<\ldots$ be a sequence of prime numbers. Denote

$$
Y_{n}=\left\{1 / p_{n}^{i+1}: i \in \omega\right\}, \quad Y_{n}^{\prime}=\{0\} \cup Y_{n} \subset \mathbb{I}=[0,1] .
$$

For the prime numbers $p_{n}, p_{k}$ we have $p_{n}^{i+1}=p_{k}^{j+1}$ iff $p_{k}=p_{n}$. Hence $Y_{n}^{\prime} \cap Y_{k}^{\prime}=$ $\{0\}(n \neq k)$. Denote also:

$$
\begin{aligned}
X_{n}^{1} & =Y_{n}^{\prime} \times\{n+1\}, \quad X_{n}^{2}=Y_{n} \times\{1 /(n+1)\}, \\
X & =\{0,0\} \cup \bigcup\left\{X_{n}^{1} \cup X_{n}^{2}: n \in \omega\right\} \subset \mathbb{I} \times \mathbb{R}, \quad Y=\bigcup\left\{Y_{n}^{\prime}: n \in \omega\right\} .
\end{aligned}
$$

Let $\pi: \mathbb{I} \times \mathbb{R} \rightarrow Y$ be the projection onto the first coordinate and $f=\pi \mid X$.

1. It is easy to see that $f$ is stable (define $\eta_{y}$ as a family of all open in $X$ subsets $U$ such that $y \in f(U)$, if $y \neq 0$ and $U \supset f^{-1}(0)$, if $\left.y=0\right)$.

2. If $f$ is an $s$-covering map, then there is a compact $X_{0} \subset X$ for which $f\left(X_{0}\right)=$ $Y$ and $X_{0} \cap X_{n}^{1} \neq \varnothing$ (since $X_{n}^{1}$ are open and closed in $X$ ) for a finite number of $n$ only. Then there is an $n$ for which the set $X_{n}^{2} \cap X_{0}$ has cardinality $\aleph_{0}$. Since $X_{n}^{2}$ is closed in $X, X_{n}^{2} \cap X_{0}$ has an accumulation point in $X_{n}^{2}$. This is impossible.

3. Define $f_{1}$ by factorising $f^{-1}(0)$ to one point $z_{0}$. Then $f_{1}$ is a closed map. The map $f_{2}$ from $Z$ onto $Y$ is defined as follows: $f_{2}(z)=0$ if $z=z_{0}$, and $f_{2}(z)=$ $f\left(f_{1}^{-1}(z)\right)$ if $z \neq z_{0}$. Then $f_{2}$ is an open map and $f=f_{2} \circ f_{1}$.

The product of two stable ( even two transquotient ) maps cannot be stable. Indeed, let us consider the product $t=f \times i d_{\mathbb{I}}: X \times \mathbb{I} \rightarrow Y \times \mathbb{I}$ of the stable, transquotient map $f$ from Example 2 and identity map $i d_{\mathbb{I}}$. If $t$ is stable, then by Theorem 0 there is a countable set $Y_{\sigma} \subset Y \times \mathbb{I}$ and $X_{0} \subset X \times \mathbb{I}$ such that $t \mid X_{0}$ is a perfect map onto $(Y \times \mathbb{I}) \backslash Y_{\sigma}$. Hence, there is $x_{0} \in \mathbb{I}$ such that $\left(Y \times\left\{x_{0}\right\}\right) \cap Y_{\sigma}=\varnothing$ and $t \mid t^{-1}\left(Y \times\left\{x_{0}\right)\right\}=f: X \rightarrow Y$ is $s$-covering. This is impossible.

\section{REFERENCES}

1. G. Choquet, Lectures on Analysis, Volume I, Math. Lecture Notes Series-W.A. Benjamin, 1969. MR 40:3252

2. G. Debs and J.Saint Raymond, Compact Covering and Game Determinacy, Topology and Appl. 68 (1996), 153-185. MR 96m:54067

3. E. Michael, Complete spaces and tri-quotient maps, Illinois J. Math. 21(3) (1977), 716-733. MR 57:7543

4. E. Michael, Partition-complete spaces and their preservation by tri-quotient and related maps, Top. and Appl. 73 (1996), 121-131. MR 97h:54016

5. A. Ostrovsky, On compact-covering mappings, Soviet Math.Dokl. 17 (1976), 606-610.

6. A. Ostrovsky, New classes of maps related to k-covering maps, Moskow University Mathematics Bulletin 49 (1994), 20-23.

7. W. Sierpiński, Sur une propriété des ensembles $G_{\delta}$, Fund. Math. (1930), 173-180.

8. A.H. Stone, Metrizability of decomposition spaces, PAMS 7 (1956), 690-700. MR 19:299b

9. I.A. Vainstein, On closed mappings of metric spaces, Dokl.Acad.Nauk USSR 57 (1947), 319321.

EDV-Büro Wenninger, Schatzbogen 58, 81829 Muenchen, Germany

E-mail address: ostrovsk@cip.mathematik.uni-muenchen.de

${ }^{3} 2$ ) was remarked by V. Popov. Note that $f$ is not a triquotient map. 